\title{
Remote CBT for Psychosis During the COVID-19 Pandemic: Challenges and Opportunities
}

\author{
Sarah L. Kopelovich ${ }^{1}$ (1) . Doug Turkington ${ }^{2}$
}

Received: 19 April 2020 / Accepted: 25 September 2020 / Published online: 1 October 2020

(c) Springer Science+Business Media, LLC, part of Springer Nature 2020

\begin{abstract}
The COVID pandemic is now leading to the emergence of a secondary mental health pandemic. Clients with psychosis are at increased risk of poorer medium- and long-term psychosocial and clinical outcomes. In response to the pressing need to flexibly deliver high-quality care to individuals with psychosis, this brief report proposes high yield cognitive behavioral techniques for psychosis (HY-CBt-p) facilitated by task sharing and digital enhancements. HY-CBt-p is delivered over fewer sessions than formulation-based Cognitive Behavioral Therapy for psychosis (CBTp), can be learned by a range of providers, and includes techniques such as developing a normalizing explanation; techniques to reduce anxiety, depression, and insomnia, which perpetuate psychotic symptoms; self-monitoring; reality testing; and wellness planning. Previous research suggests that effect sizes will be lower than that of 16-session formulation-driven CBTp, but additional research is needed to test the feasibility, acceptability, efficacy, and comparative effectiveness of different forms of remote-delivered CBTp.
\end{abstract}

Keywords Cognitive behavioral therapy for psychosis · Serious mental illness · Telehealth · COVID-19

Individuals with serious mental illness (SMI) are particularly vulnerable during public health crises such as the COVID19 global pandemic (Druss, 2020). In a recent rapid review of the literature, Brown and colleagues (2020) suggest that the very public health measures that are intended to keep individuals safe from contracting COVID-19 may be exacerbating psychotic symptoms among those who have a preexisting psychotic diagnosis, and may also increase the risk of developing a new-onset psychotic episode among those who do not. This trend parallels that which we are witnessing among individuals with substance use, anxiety, and depression (Meadows Mental Health Policy Institute (2020). In light of these unprecedented circumstances, frontline providers have had to extemporize and adapt in-person services to meet the needs of their clients with SMI. This brief report is intended to advise community mental health practitioners in

Sarah L. Kopelovich

skopelov@uw.edu

1 Department of Psychiatry and Behavioral Sciences, Harborview Medical Center, University of Washington, 325 Ninth Avenue, Box 359911, Seattle, WA 98104, USA

2 Cumbria Northumberland Tyne and Wear NHS Foundation Trust, Monkwearmouth Hospital, Newcastle Road, Tyne and Wear, Sunderland, UK cognitive behavioral interventions for psychotic symptoms that are relatively easy to learn, to coach others (e.g., natural supports) in, and that are amenable to telephonic encounters, telehealth, or digital accompaniment. Similar explorations of practice considerations for CBT delivery during the pandemic have been published for other client populations (e.g., Waller et al. 2020), but, to our knowledge, this is the first such paper that details high-yield strategies and key considerations for remote or hybridized care delivery for clients with psychosis. The recommendations provided in this brief report are based on the authors' experiences in CBT for psychosis (CBTp) protocol development, training practitioners and families in these techniques; and technology-assisted CBTp service delivery and implementation.

Cognitive behavioral interventions should be considered a frontline intervention during this critical time for three key reasons. First, CBT is a transdiagnostic treatment that has become the dominant psychological approach to treating the disorders that are considered serious mental illnesses, as well as substance use disorders and suicidality (NICE 2014; APA 2004). The CBT model, framework, and skills can therefore be flexibly applied to a broad range of preexisting and emerging diagnoses and concerns, lending further flexibility during an unpredictable period. Furthermore, research suggests that certain transdiagnostic high-yield 
cognitive-behavioral interventions and CBTp guided selfhelp can provide symptomatic relief, enhance treatment adherence and engagement, and sustain therapeutic gains (Naeem et al. 2016a, 2016b; Malik et al. 2009; Wright et al. 2010). High-yield cognitive behavioral techniques for psychosis (HY-CBt-p) are "key CBT methods" that are easily learned and deliver significant symptomatic improvement without the structure and process of a formal course of therapy (Wright et al. 2010, p. xv). These techniques can be implemented along with self-help guides (e.g., the Overcoming book series, which guides readers through a brief formulation and key CBT techniques for the presenting problem) or mobile mental health applications (discussed further, below), which are inexpensive, easy to use, and can penetrate difficult-to-access populations (Naeem et al. 2016a). HY-CBt-p and guided self-help strategies include psychoeducation and normalization, self-monitoring, cognitive and behavioral coping skills, reality testing, and developing a relapse prevention plan. These can be taught in brief clinical encounters, and are therefore suitable to telephonic and telehealth sessions, which are susceptible to interruptions caused by poor connection, lack of privacy in the client's environment, and environmental distractions. While they maintain the structural components of a traditional CBT session, HY-CBt-p sessions target emerging, short-term concerns, and are typically provided in fewer than the $16+$ sessions characteristic of formulation-based CBTp. Clients may see benefits in relapse delay and prevention in as few as six sessions (Malik et al. 2009).

Second, unlike formulation-based CBTp, HY-CBt-p and guided self-help cognitive and behavioral interventions can be easily learned by a range of behavioral health professionals, paraprofessionals, and non-professionals (Garety et al. 2018; Turkington et al. 2002; Waller et al. 2014). In the U.S., case managers were able to safely deliver HY-CBt-p, resulting in significant improvements in overall symptoms, depression, hallucinations, and negative symptoms following 12 sessions (Turkington et al. 2014). Natural supports who received a multiday workshop in CBTp principles and high-yield techniques have also demonstrated good uptake of CBTp skills and report more therapeutic involvement in their loved ones' recoveries (Turkington et al. 2018; Kopelovich et al. 2019). Global organizations like the World Health Organizations recommend task shifting or task sharing approaches like these not just as a stop-gap during times of need, but as a sustainable approach to population-based mental health care (Lancet 2019). Practitioners who are not familiar with CBTp principles or strategies may find remote training through organizations like the North American CBT for Psychosis Network (www.nacbtp.org) or the Beck Institute (www.beckinstitute.org). Practitioners already versed in cognitive and behavioral interventions for secondary symptoms such as anxiety, depression, and insomnia can apply these interventions to their clients with psychosis, as evidence suggests that negative affect, negative self-schemas, and sleep disruptions are key perpetuators of psychotic symptoms (Reeve et al. 2018).

Finally, cognitive behavioral interventions are highly adaptable to our current state of remote care. CBT is frequently delivered through or enhanced by remote applications, including telepsychiatry (Hartley et al. 2014), as well as smartphone applications (Ben-Zeev et al. 2014a, b), wearable devices (Naslund et al. 2016), texting (Ben-Zeev et al. 2014a, b), web-based platforms (Gottlieb et al. 2013), and virtual reality (Freeman et al. 2016). In fact, emerging evidence suggests that e-mental health interventions-which can facilitate expanded healthcare reach, improved clinical decision-making, improved management of chronic health conditions, and sustained clinical contact during emergencies (Varshney 2014) - are not only palatable to clients with psychosis (Daker-White and Rogers 2013; SantestebanEcharri et al. 2020), but may be preferable to co-located service delivery (Bee et al. 2010). The therapeutic effects of teletherapy and human-assisted mHealth interventions for clients with SMI appear to rival that of clinic-based treatment (Backhaus et al. 2012; Ben-Zeev et al. 2018). Given that individuals with psychosis are more likely to have a smartphone than a computer or other device (Aschbrenner et al. 2018), CBT apps applicable to clients with SMI should be integrated into skills coaching (for a recent review, see Torous and Keshavan 2020). This requires the HY-CBt-p provider to possess a facility with these mHealth interventions and for the provider to spend some session time providing coaching and troubleshooting on consistent use of these apps (Wilhelm and Jacobson 2020). While reciprocal applications, in which clinicians are able to access and integrate client-supplied data for symptom surveillance, relapse mitigation, and treatment integration, do exist, they are not yet mainstream (Ben-Zeev et al. 2019).

\section{Remote Delivery Considerations for HY-CBt-p}

While these developments are encouraging, we cannot wait for promising practices to manifest in everyday clinical practice. During the pandemic, clinicians will need to utilize currently available approaches and develop creative and pragmatic adaptations to facilitate cognitive and behavioral change. These include in- and between-session strategies to promote learning and rehearsal of cognitive behavioral change strategies. Examples of in-session strategies include creating PowerPoint presentations of common topics covered during CBT sessions to enable multimodal learning; use of the Whiteboard feature in the video platform; screensharing for fillable pdf-worksheets, handouts, websites, 
videos, and data dashboards; bringing the natural support person-of-choice into the session to facilitate co-learning; sending secure post-session communication of key points and action plans; and sending therapy or CBT self-help resources through the physical mail. Between-session support strategies to enhance recall and skill rehearsal include behavioral tailoring for medication consistency; commercially-available smartphone applications that can automate prompts for activity scheduling; calendar reminders for home practice assignments; accessing peer support bulletin boards and virtual support groups; traditional bibliotherapy and/or assigning CBT-informed podcast episodes; telephone support; and use of short message service (SMS) to prompt behavior (Bell et al. 2017). Although empirical evidence of the integration of modalities and strategies among clients with psychosis is lacking, they have intuitive appeal as a means of optimizing therapeutic benefit of remote-delivered CBT, HY-CBt-p, and guided self-help, as well as in maximizing efficiency. For instance, a clinician with a large caseload of clients with psychosis who are able to engage in telehealth could create brief PowerPoint slides to correspond to HY-CBt-p concepts and techniques, and which reference corresponding worksheets. These can then be used across clients, clinicians, and modalities (e.g., individual and group therapy; in-person and teletherapy).

Both practitioners and clients confront practical and psychological challenges to all-remote or a remote- and inperson hybridized model. Practical challenges include lack of access to the hardware, software, or data plans required for telepsychiatry; poor connections that disrupt the flow of a therapeutic encounter; and delays in the exchange of written therapeutic materials. Practitioners may also experience administrative burdens associated with revised workflows, policies, and procedures. Clients may not yet be facile enough with the technology to shift care to these methods, or there may be concerns about the clients' ability to secure a private location for session. Psychological challenges include discomfort among both service users and providers with these treatment modalities, the difficulty of making the transition and adapting to remote administration as either the sole or primary encounter type, worry thoughts related to technology and surveillance, or a feeling of unreality associated with virtual encounters. Practitioners may need to engage in befriending sessions with new clients or those who are experiencing an increase in persecutory ideation. During befriending sessions, the focus should be on enjoyable and emotionally neutral topics. Practitioners should be mindful of delusional elaboration during telehealth sessions among clients with paranoid tendencies. Audio-only delivery may pose challenges with some clients, as body language and mood shifts are much more difficult to assess. As such, working with automatic thoughts and generating rational responses can be hindered. Finally, given the prevalence of cognitive impairments among individuals with psychotic disorders and the taxing nature of sustained attention during phone or video-based sessions, shorter, more frequent sessions is recommended.

\section{Special Considerations for Clients with Cognitive Impairment}

There is little empirical guidance about how to design and implement remote or hybridized CBT interventions to address the accessibility needs of clients with cognitive deficits. Likewise, user interfaces for digital health platforms are rarely if ever designed for clients with cognitive impairments (Rotondi 2014). Agencies can better support these clients by providing educational and behavioral supports like information tip sheets with large graphic images, offering technology learning sessions, and including natural supports during sessions. The manner and extent to which cognitive impairments will affect clients' response to remote CBT interventions will depend on the nature and severity of existing cognitive impairment and cognitive vulnerabilities. Unfortunately, few community mental health clinicians are trained to assess cognitive impairments in a way that enables a nuanced understanding, and many are similarly often unaware of or trained in the modifications or treatments indicated to address cognitive challenges among adults with SMI. Whereas mobile- (e.g., wearable technology, smartphones), hybrid- (e.g., tablets, telehealth), and homebased- (e.g., video games, smart home) devices are emerging as avenues for both intermittent and continuous cognitive assessment and intervention, these remain in nascent stages of development and testing as of this writing (for a review, see Hays et al. 2019).

\section{Discussion}

The COVID-19 pandemic represents a protracted communal stressor that is expected to affect the content, incidence, and severity of psychotic symptoms, both among those who have and those who are at risk of developing a psychotic disorder (Brown et al. 2020). Given that individuals' psychiatric symptoms, substance use disorders, and environmental circumstances place clients with psychosis at higher risk of adverse social, physical, and mental health outcomes (Druss 2020), community mental health practitioners need to consider strategies that permit greater penetration of evidence-based interventions that are amenable to all-remote or hybridized delivery modes during the pandemic. Strategies to scale-up evidence-based interventions include task sharing, mobile health, and technique-based or guided selfhelp interventions (Kola 2020). This brief report synthesizes 
these suggestions by recommending that HY-CBt-p, offered by a range of providers and supported through digital platforms and natural supports, can help to maximize efficiency and penetration of empirically-supported care practices for clients with psychosis. That said, the authors acknowledge both the pragmatic limitations of this approach (e.g., insufficiently trained workforce, lack of technological infrastructure across large regions of both high- and low-income countries) and the empirical limitations. With regard to the latter, while there is some evidence to suggest that tele-CBTp interventions are palatable, if not preferable to clients with psychosis (Santesteban-Echarri et al. 2020), and to suggest that brief or technique-based CBTp interventions are more effective at addressing psychotic-related distress than treatment as usual (Naeem et al. 2016b), more research is needed to evaluate the effectiveness of "high-yield" strategies and to ascertain the comparative effectiveness of HY-CBt-p and formulation-based CBTp conducted via telehealth. If mental health systems around the world are able to serve clients with psychosis in the ways suggested in this brief report-namely, by increasing access to CBTp interventions and actualizing decades-old recommendations to incorporate natural supports and digital technology to support clinical care-we may be able to both mitigate the effects of pandemic-related stress on individuals with psychosis and emerge from this crisis with a system that leverages twenty-first century advances in health and technology for this population.

\section{Compliance with Ethical Standards}

Conflict of interest The authors have no conflicts of interest to disclose and all authors certify responsibility for the content of this article.

Research Involving Human and Animal Rights This work does report on research involving human participants or animal subjects and did not require ethical approval from an Institutional Review Board.

\section{References}

American Psychiatric Association. (2004). Practice guideline for the treatment of patients with schizophrenia. Retrieved August 4, 2020, from https://psychiatryonline.org/pb/assets/raw/sitewide/ practice_guidelines/.

Aschbrenner, K. A., Naslund, J. A., Grinley, T., Bienvenida, J. C., Bartels, S. J., \& Brunette, M. (2018). A survey of online and mobile technology use at peer support agencies. Psychiatry, 4, 1-10.

Backhaus, A., Agha, Z., Maglione, M. L., Repp, A., Ross, B., Zuest, D., et al. (2012). Videoconferencing psychotherapy: A systematic review. Psychological Services, 9(2), 111-131. https://doi. org/10.1037/a0027924.

Bee, P. E., Lovell, K., Lidbetter, N., Easton, K., \& Gask, L. (2010). You can't get anything perfect: User perspectives on the delivery of cognitive behavioural therapy by telephone. Social Science and Medicine, 71(7), 1308-1315.

Bell, I. H., Lim, M. H., Rossell, S. L., \& Thomas, N. (2017). Ecological momentary assessment and intervention in the treatment of psychotic disorders: A systematic review. Psychiatric Services, 68(11), 1172-1181. https://doi.org/10.1176/appi.ps.201600523.

Ben-Zeev, D., Brenner, C. J., Begale, M., Duffecy, J., Mohr, D. C., \& Mueser, K. T. (2014a). Feasibility, acceptability, and preliminary efficacy of a smartphone intervention for schizophrenia. Schizophrenia Bulletin, 40(6), 1244-1253. https://doi. org/10.1093/schbul/sbu033.

Ben-Zeev, D., Buck, B., Kopelovich, S., \& Meller, S. (2019). A technology-assisted life of recovery from psychosis. NPJ Schizophrenia, 5(1), 1-4.

Ben-Zeev, D., Brian, R. M., Jonathan, G., Razzano, L., Pashka, N., Carpenter-Song, E., et al. (2018). Mobile health (mHealth) versus clinic-based group intervention for people with serious mental illness: A randomized controlled trial. Psychiatric Services, 69(9), 978-985. https://doi.org/10.1176/appi.ps.201800063.

Ben-Zeev, D., Kaiser, S. M., \& Krzos, I. (2014b). Remote "hovering" with individuals with psychotic disorders and substance use: Feasibility, engagement, and therapeutic alliance with a textmessaging mobile interventionist. Journal of Dual Diagnosis, 10(4), 197-203

Brown, E., Gray, R., Lo Monaco, S., O’Donoghue, B., Nelson, B., Thompson, A., et al. (2020). The potential impact of COVID19 on psychosis: A rapid review of contemporary epidemic and pandemic research. Schizophrenia Research. https://doi. org/10.1016/j.schres.2020.05.005.

Daker-White, G., \& Rogers, A. (2013). What is the potential for social networks and support to enhance future telehealth interventions for people with a diagnosis of schizophrenia: A critical interpretive synthesis. BMC Psychiatry, 13(1), 279. https://doi. org/10.1186/1471-244X-13-279.

Druss, B. G. (2020). Addressing the COVID-19 pandemic in populations with serious mental illness. JAMA Psychiatry. https://doi. org/10.1001/jamapsychiatry.2020.0894.

Freeman, D., Bradley, J., Antley, A., Bourke, E., DeWeever, N., Evans, N., et al. (2016). Virtual reality in the treatment of persecutory delusions: randomised controlled experimental study testing how to reduce delusional conviction. The British Journal of Psychiatry, 209(1), 62-67.

Garety, P. A., Craig, T. K., Iredale, C. H., Basit, N., FornellsAmbrojo, M., Halkoree, R., et al. (2018). Training the frontline workforce to deliver evidence-based therapy to people with psychosis: Challenges in the GOALS study. Psychiatric services, 69(1), 9-11.

Gottlieb, J. D., Romeo, K. H., Penn, D. L., Mueser, K. T., \& Chiko, B. P. (2013). Web-based cognitive-behavioral therapy for auditory hallucinations in persons with psychosis. Schizophrenia Research, $145,82-87$

Hartley, S., Scarratt, P., Bucci, S., Kelly, J., Mulligan, J., Neil, S. T., et al. (2014). Assessing therapist adherence to recovery-focused cognitive behavioural therapy for psychosis delivered by telephone with support from a self-help guide: Psychometric evaluations of a new fidelity scale. Behavioural and cognitive Psychotherapy, 42(4), 435-451.

Hays, R., Henson, P., Wisniewski, H., Hendel, V., Vaidyam, A., \& Torous, J. (2019). Assessing cognition outside of the clinic: Smartphones and sensors for cognitive assessment across diverse psychiatric disorders. Psychiatric Clinics, 42(4), 611-625.

Kola, L. (2020). Global mental health and COVID-19. Lancet Psychiatry. https://doi.org/10.24869/psyd.2020.6.

Kopelovich, S., Stiles, B., Monroe-DeVita, M., Hardy, K., Davis, S., Turkington, D. (2019). Training family and caregivers in CBT for psychosis-Informed skills within the context of a CBTp provider 
network. Symposium at the association for behavioral and cognitive therapies, 52nd Annual Meeting, Atlanta, GA.

Lancet,. (2019). Task sharing: Stopgap or end goal? The Lancet Psychiatry, 6(2), 81. https://doi.org/10.1016/S2215-0366(19)30006-9.

Malik, N., Kingdon, D., Pelton, J., Mehta, R., \& Turkington, D. (2009). Effectiveness of brief cognitive-behavioral therapy for schizophrenia delivered by mental health nurses: Relapse and recovery at 24 months. The Journal of Clinical Psychiatry, 70(2), 201-207. https ://doi.org/10.4088/JCP.07m03990.

Meadows Mental Health Policy Institute (2020). COVID-19 Response Briefing: Mental Health and Substance Use Disorder Impacts of a COVID-19 Economic Recession. Retrieved August 4, 2020, from https://www.texasstateofmind.org/uploads/whitepapers/COVID -MHSUDImpacts.pdf

Naeem, F., Johal, R., McKenna, C., Rathod, S., Ayub, M., Lecomte, T., et al. (2016a). Cognitive behavior therapy for psychosis based guided self-help (CBTp-GSH) delivered by frontline mental health professionals: Results of a feasibility study. Schizophrenia Research, 173, 69-74. https://doi.org/10.1016/j.schre s.2016.03.003.

Naeem, F., Khoury, B., Munshi, T., Ayub, M., Lecomte, T., Kingdon, D., et al. (2016b). Brief cognitive behavioral therapy for psychosis (CBTp) for schizophrenia: Literature review and meta-analysis. International Journal of Cognitive Therapy, 9(1), 73-86.

Naslund, J. A., Aschbrenner, K. A., \& Bartels, S. J. (2016). Wearable devices and smartphones for activity tracking among people with serious mental illness. Mental Health and Physical Activity, 10, 10-17. https://doi.org/10.1016/j.mhpa.2016.02.001.

National Institute for Clinical Excellence. (2014). Psychosis and schizophrenia in adults: Prevention and management (Guideline No. 178). Retrieved April 15, 2020, from https://www.nice.org.uk/ guidance/cg178/chapter/1-Recommendations/promoting-recov ery-and-possible-future-care- 2 .

Reeve, S., Nickless, A., Sheaves, B., \& Freeman, D. (2018). Insomnia, negative affect, and psychotic experiences: Modelling pathways over time in a clinical observational study. Psychiatry Research, 269, 673-680. https://doi.org/10.1016/j.psychres.2018.08.090.

Rotondi, A. (2014). Schizophrenia. In M. A. Cucciare \& K. R. Weingardt (Eds.), Using technology to support evidence-based behavioral health practices: A clinician's guide (pp. 69-90). Abingdon UK: Routledge.

Santesteban-Echarri, O., Piskulic, D., Nyman, R. K., \& Addington, J. (2020). Telehealth interventions for schizophrenia-spectrum disorders and clinical high-risk for psychosis individuals: A scoping review. Journal of Telemedicine and Telecare, 26(1-2), 14-20. https://doi.org/10.1177/1357633X18794100.
Torous, J., \& Keshavan, M. (2020). COVID-19, mobile health and serious mental illness. Schizophrenia Research. https://doi. org/10.1016/j.schres.2020.04.013.

Turkington, D., Gega, L., Lebert, L., Douglas-Bailey, M., Rustom, N., Alberti, M., et al. (2018). A training model for relatives and friends in cognitive behaviour therapy (CBT) informed care for psychosis. Cogent Psychology. https://doi.org/10.1080/23311 908.2018.1497749.

Turkington, D., Kingdon, D., \& Turner, T. (2002). Effectiveness of a brief cognitive-behavioural therapy intervention in the treatment of schizophrenia. The British Journal of Psychiatry, 180(6), 523-527.

Turkington, D., Munetz, M., Pelton, J., Montesano, V., Sivec, H., Nausheen, B., et al. (2014). High-yield cognitive behavioral techniques for psychosis delivered by case managers to their clients with persistent psychotic symptoms: An exploratory trial. Journal of Nervous and Mental Disease, 202(1), 30-34. https://doi. org/10.1097/NMD.0000000000000070.

Varshney, U. (2014). Mobile health: Four emerging themes of research. Decision Support Systems, 66, 20-35. https://doi.org/10.1016/j. dss.2014.06.001.

Waller, G., Pugh, M., Mulkens, S., Moore, E., Mountford, V. A., Carter, J., et al. (2020). Cognitive-behavioral therapy in the time of coronavirus: Clinician tips for working with eating disorders via telehealth when face-to-face meetings are not possible. International Journal of Eating Disorders. https://doi.org/10.1002/eat.23289.

Waller, H., Craig, T., Landau, S. et al. (2014). The effects of a brief CBT intervention, delivered by frontline mental health staff, to promote recovery inpeople with psychosis and comorbid anxiety or depression (the GOALS study): Study protocol for a randomized controlled trial. Trials 15, 255.https://doi. org/10.1186/1745-6215-15-255.

Wilhelm, S., \& Jacobson, N. (2020). Cognitive-behavioral therapy in the digital age: Presidential address. Behavior Therapy, 51(1), 1-14. https://doi.org/10.1016/j.beth.2019.08.001.

Wright, J., Sudak, D., Turkington, D., \& Thase, M. (2010). High-yield cognitive behavior therapy for brief sessions: An illustrated guide. Washington, DC: American Psychiatric Publishing Inc.

Publisher's Note Springer Nature remains neutral with regard to jurisdictional claims in published maps and institutional affiliations. 